CLINICAL STUDY

\title{
Bone turnover and metabolism in paediatric patients with inflammatory bowel disease treated with systemic glucocorticoids
}

\author{
Marianne K Vihinen, Kaija-Leena Kolho, Merja Ashorn ${ }^{1}$, Matti Verkasalo and Taneli Raivio \\ Hospital for Children and Adolescents, Helsinki University Central Hospital, University of Helsinki, Helsinki, Finland and ${ }^{1}$ Department of Paediatrics, \\ University of Tampere, Tampere, Finland
}

(Correspondence should be addressed to M Vihinen, Biomedicum Helsinki 2 C, PO Box 705, 00029 HUCH, Helsinki, Finland;

Email: marianne.vihinen@helsinki.fi)

\begin{abstract}
Objective: We investigated circulating markers of bone turnover before and during systemic glucocorticoid treatment in paediatric patients with inflammatory bowel disease (IBD).

Methods: Twenty-two children (mean age, 12.3 years) with IBD necessitating peroral steroid therapy were studied, with special reference to bone formation and resorption markers amino-terminal type I collagen propeptide (PINP) and carboxyterminal telopeptide of type I collagen (ICTP) respectively. In addition, GH-related IGF-I and sex hormone-binding protein (SHBG) were measured. Bone markers were analyzed at the initiation of the glucocorticoid treatment, at 2 and 5 weeks thereafter and at 1 month following the withdrawal of the steroid. Control group comprised 22 IBD patients in remission. Results: PINP and IGF-I were already lower before glucocorticoid treatment serum in children with active IBD as compared with control children with IBD in remission (median PINP $271 \mathrm{vs} 535 \mu \mathrm{g} / \mathrm{l}$, $P<0.05$; IGF-I 23 vs $29 \mathrm{nmol} / \mathrm{l}, P<0.05)$. After 2 weeks of glucocorticoid treatment serum PINP levels had declined further, from 271 to $163 \mu \mathrm{g} / \mathrm{l}(P<0.001)$, serum ICTP from 14.2 to $9.6 \mu \mathrm{g} / \mathrm{l}$ $(P<0.001)$, and SHBG from 54 to $35 \mathrm{nmol} / \mathrm{l}(P<0.001)$ respectively. By contrast, serum IGF-I increased from 23 to $37 \mathrm{nmol} / \mathrm{l}(P<0.001)$. One month after the withdrawal of the glucocorticoid, all bone markers restored to levels similar to the controls.

Conclusions: Bone formation in children with active IBD appears compromised and systemic glucocorticoid treatment further suppresses bone turnover. After the cessation of the glucocorticoid the bone markers show immediate improvement.
\end{abstract}

European Journal of Endocrinology 159 693-698

\section{Introduction}

Glucocorticoids have been an established part of the medical treatment of inflammatory bowel disease (IBD) since the 1950's, when it was first realized that these drugs improve survival (1). Since the early days, the clinical practice in glucocorticoid therapy has remained much the same (2). It is estimated that $60-80 \%$ of IBD patients benefit from the treatment, but the factors leading to inadequate response are poorly understood $(3,4)$. Consequently, there are no means to predict the probable response of an individual in advance. Glucocorticoid responsiveness may be genetically determined but the exact molecular mechanisms behind the development of harmful side effects are still obscure $(5,6)$. The most common side effects include sleep disturbances, development of moon-face, abdominal distention, growth arrest and decrease in bone mineral density (6). Occasionally vertebral fracture may appear, resulting from high glucocorticoid exposure, increased sensitivity to administered steroids or both (7).
The effects glucocorticoid treatment and the underlying disease have on bones can be assessed indirectly by measuring the circulating markers of bone turnover and metabolism $(8,9)$. The formation of type I collagen, the main component of bone organic matrix, is reflected in serum levels of amino-terminal (PINP) and carboxyterminal type I procollagen propeptides that are cleaved from both ends of the molecule $(10,11)$. The carboxyterminal telopeptide of type I collagen (ICTP), instead, is a degradation product of type I collagen and as such measures bone resorption (12).

Growth hormone $(\mathrm{GH})$ is an important determinant of bone health in paediatric patients. GH exerts its effects both directly and through insulin-like growth factors I (IGF-I) and II and the IGF binding proteins 1-6 (13, 14). Circulating IGF-I has an important role in the acquisition of peak bone mass and the GH-IGF system is vital for optimal bone accrual and linear growth (15). In humans, GH also indirectly regulates circulating levels of sex hormone-binding protein (SHBG), the transporter of sex hormones (16). However, SHBG also 
acts as an independent predictor of bone turnover rate in adults (17). In children, the relationship between SHBG and bone turnover has not been addressed.

In this study, we assessed the effect of glucocorticoid intake on acute changes in bone formation and resorption markers in parallel with the markers of inflammation in paediatric patients with IBD.

\section{Materials and methods}

\section{Subjects}

The study group comprised 22 children and adolescents with IBD (mean age, 12.3 years; age range, 3.6-18 years, Table 1), necessitating peroral steroid therapy due to the acute exacerbation of the disease. The children had previously undergone gastrointestinal endoscopies and had their diagnosis settled according to the Lennard-Jones criteria (18). They were prospectively enrolled to the study from the Outpatient Clinics for Paediatric Gastroenterology at the University Hospitals of Helsinki and Tampere. Inclusion criteria were age $\leq 18$ years, no steroid therapy for at least 1 month prior to the study. The glucocorticoid bioactivity (GBA) in these patients has been published $(19,20)$.

The treatment was started with peroral prednisolone ( $1 \mathrm{mg} / \mathrm{kg}$ per $\mathrm{d}, n=19)$ or budesonide $(9 \mathrm{mg} / \mathrm{d}, n=3$ ) according to the clinician's decision. During the course of the therapy, the dose was tapered according to standard clinical practice. The patients were clinically examined at the start of the study, at 2 and 5 weeks after starting the steroid, and at 1 month after the cessation of the glucocorticoid. Therapeutic response was defined according to clinicians' judgment of clinical improvement (decrease in stool rate and the amount of blood in the stools as well as abating diarrhoea) and decrease in the inflammatory markers during the first 2 weeks of steroid therapy. Special attention was paid to acute (appearing

Table 1 The clinical characteristics of the 22 patients with inflammatory bowel disease (IBD) and their respective controls.

\begin{tabular}{llll}
\hline & Patients & Controls & $\boldsymbol{P}$ \\
\hline UC & $n=19$ & $n=18$ & \\
CD & $n=3$ & $n=4$ & \\
Age (median, & $13.5(3.6-18)$ & $13.9(4.4-17.6)$ & NS \\
range, years) & & & \\
Sex & $n=13$ & $n=13$ & \\
$\quad$ Boys & $n=9$ & $n=9$ & \\
Girls & & $n=8$ & \\
Tanner & $n=7$ & $n=5$ & NS \\
$\begin{array}{l}\text { Stage I } \\
\text { Stages II and III }\end{array}$ & $n=5$ & $n=9$ & \\
$\begin{array}{l}\text { Stages IV and V } \\
\text { BMI (median, }\end{array}$ & $17.1(13.3-22.6)$ & $18.9(14.3-24.8)$ & \\
range, kg/m $\left.{ }^{2}\right)$ & & $8(3-33)$ & \\
ESR (median, & $29(6-81)$ & & \\
range, mm/h) & & & \\
\hline
\end{tabular}

UC, ulcerative colitis; CD, Crohn's disease; BMI, body mass index; ESR, erythrocyte sedimentation rate. during the first month of the treatment) glucocorticoidrelated side effects: weight gain, development of moonface, striae or acne. The development of side effects and therapeutic response were both graded with a two-grade scale (yes/no side effects, good/poor response). Three patients with Crohn's disease (CD) were included in the study, for they presented primarily with colitis. Before glucocorticoid treatment, 16 patients received 5-aminosalicylic acid (5-ASA), two patients both azathioprine and 5-ASA, one patient antibiotics and 5-ASA, one patient only antibiotics and two had no medication due to hypersensitivity reactions.

\section{Control group}

Twenty-two age, sex, pubertal maturation and disease subtype matched children and adolescents with IBD in remission comprised the control group (Table 1). None of these children had been on glucocorticoid therapy during the preceding 2 months at minimum. Their maintenance medication was similar to that in the patient group: 17 controls received 5-ASA, three patients received both azathioprine and 5-ASA and two had no medication due to adverse reactions.

\section{Laboratory analyses}

At the start of the glucocorticoid therapy, at 2 and 5 weeks thereafter, and at 1 month after discontinuing the glucocorticoid, a venous blood sample was drawn between 1100 and $1500 \mathrm{~h}$. The analyses carried out in a clinical laboratory included blood count, blood haemoglobin concentration and erythrocyte sedimentation rate (ESR). Serum N-terminal propeptide of type I procollagen (PINP) and C-terminal telopeptide of type I collagen (ICTP) were measured with UniQ radioimmunoassays (Orion Diagnostica Oy, Espoo, Finland) that have intra- and interassay coefficients of variation $(\mathrm{CV})$ of $<10.2 \%$ for PINP and $<9.4 \%$ for ICTP respectively. Serum sex-hormone binding globulin (SHBG) was quantified with a time-resolved immunofluorometric assay (AutoDELFIA, Wallac, Turku, Finland) with intraassay $\mathrm{CV}$ of $<1.8 \%$ and interassay $\mathrm{CV}$ of $<10.1 \%$. Serum IGF-I was measured with an immunochemiluminometric assay on the IMMULITE 2000 analyzer (DPC, Los Angeles, CA, USA) with inter-assay $\mathrm{CV}$ of $<4 \%$ and total $\mathrm{CV}$ of $<9 \%$ in the range of $10-180 \mathrm{nmol} / \mathrm{l}$. Faecal calprotectin was measured with an enzyme immunoassay (Phical test; Calpro AS, Oslo, produced by NovaTec Immunodiagnostica, Dietzenbach, GmBH, Germany) as previously described (21). Serum GBA levels were obtained from previous work $(19,20)$.

\section{Statistical analysis}

Values are expressed as median (range). Mann-Whitney's $U$ test, Kruskal-Wallis test, Wilcoxon's signed rank sum 
test and Spearman's rank order correlation test were used when investigating associations between non-parametric variables. Statistical analyses were performed using SPSS by SPSS Inc. software (Chicago, Illinois, USA). $P<0.05$ was accepted to indicate statistical significance.

\section{Ethical considerations}

The families were thoroughly informed about the study, and a written informed consent was obtained from the patient and their guardians. The study protocol was approved by the ethics committee for the Hospital for Children and Adolescents, University of Helsinki and the ethics committee of Tampere University Hospital, Tampere, Finland.

\section{Results}

\section{Type I collagen markers reflecting bone formation (PINP) and resorption (ICTP)}

In children with active IBD, the median serum PINP before glucocorticoid treatment was significantly lower than in the control children with IBD in remission (for values, see Table 2). Serum PINP levels declined by $40 \%$ already at 2 weeks after glucocorticoid therapy (Table 2). One month after the cessation of the steroid the PINP levels were significantly higher than the pretreatment levels in active IBD and similar to the levels of the children with IBD in remission (Table 2). In active IBD, serum PINP correlated with serum ICTP (before glucocorticoid therapy: $r=0.459, n=22, P<0.05)$. There was an inverse correlation with pre-treatment serum PINP and the inflammatory markers of IBD, faecal calprotectin $(r=-0.550, n=15, P<0.05)$ and ESR $(r=-0.672, n=20, P<0.01)$. During glucocorticoid treatment no correlations were found between PINP and ICTP, GBA or the inflammatory markers $(r=-0.443-0.388, P=0.091-0.705)$. The development of glucocorticoid related side effects or the response to treatment were not related to serum PINP (data not shown). Further, the PINP levels were similar between ulcerative colitis (UC; $276 \mu \mathrm{g} / \mathrm{l}, 88-849$, $n=19)$ and CD $(156 \mu \mathrm{g} / \mathrm{l}, 118-443, n=3, P=0.36)$.
PINP levels did not differ between patients at different pubertal stages (Tanner I $285 \mu \mathrm{g} / \mathrm{l}, 118-849$; Tanner II and III $296 \mu \mathrm{g} / \mathrm{l}, 200-534$; Tanner IV and V $243 \mu \mathrm{g} / \mathrm{l}$, $88-711, P=0.61)$.

The median ICTP level of the children with active IBD before glucocorticoid treatment was not significantly different from the children with IBD in remission (Table 2). During the steroid therapy, the ICTP levels declined by $40 \%$ and after therapy rose to a higher level than before treatment (Table 2). The pre-treatment ICTP levels in UC and CD patients were 14.3 (7.6-26.3, $n=19) \mu \mathrm{g} / \mathrm{l}$ and $10.8(8.7-14.1, n=3, P=0.13) \mu \mathrm{g} / \mathrm{l}$ respectively. Serum ICTP levels were not related to serum GBA levels, to the development of acute glucocorticoid related side effects, response to treatment or pubertal maturation (data not shown).

\section{Changes in serum IGF-I and SHBG}

The median IGF-I level prior to glucocorticoid therapy was significantly lower in children with active IBD than in the control children with IBD in remission (Table 2). In contrast to other markers, the levels of IGFI rose by $60 \%$ during glucocorticoid therapy and after treatment decreased to a level similar to the controls (Table 2). However, post-treatment values remained higher than before treatment. The IGF-I levels did not differ between UC and CD patients (24 vs $21 \mathrm{nmol} / \mathrm{l}$, $P=0.59)$. Pre-treatment IGF-I correlated positively with patients' weight $(r=0.805, n=22, P<0.001)$, height $(r=0.732, n=22, P<0.001)$ and BMI $(r=0.776$, $n=22, P<0.001)$. In pre-pubertal patients, the median IGF-I was $9 \mathrm{nmol} / \mathrm{l}(3-21, n=7)$, the levels rose with advancing pubertal stages (Tanner II and III $23 \mathrm{nmol} / \mathrm{l}$, 9-26, $n=5$; Tanner IV and V $31.5 \mathrm{nmol} / \mathrm{l}, 21-37$, $n=10$ respectively, $P<0.01)$. The markers of inflammation or bone metabolism, PINP and ICTP, did not correlate with serum IGF-I $(r=-0.410-0.222$, $P=0.07-0.93)$ during the study. Response to glucocorticoid treatment, acute glucocorticoid related sideeffects or serum GBA levels did not relate to serum IGF-I (data not shown).

Before the onset of the glucocorticoid therapy the median serum SHBG levels were similar between

Table 2 The markers of bone turnover and metabolism in 22 paediatric patients with active inflammatory bowel disease (IBD) treated with peroral glucocorticoids, and in 22 age- and sex-matched controls with IBD in clinical remission.

\begin{tabular}{|c|c|c|c|c|c|c|c|c|c|c|}
\hline \multirow[b]{3}{*}{ Marker } & \multicolumn{8}{|c|}{ Active IBD } & \multicolumn{2}{|l|}{ Controls } \\
\hline & \multicolumn{2}{|c|}{ Pre-treatment } & \multicolumn{2}{|l|}{2 weeks } & \multicolumn{2}{|l|}{5 weeks } & \multicolumn{2}{|c|}{ One month after treatment } & \multirow[b]{2}{*}{ Median } & \multirow[b]{2}{*}{ Range } \\
\hline & Median & Range & Median & Range & Median & Range & Median & Range & & \\
\hline S-PINP $(\mu \mathrm{g} / \mathrm{l})$ & $271^{*}$ & $88-849$ & $163^{\ddagger}$ & $29-472$ & $152^{\ddagger}$ & $23-393$ & $516^{\dagger} / \mathrm{NS}$ & 143-909 & 535 & $111-1390$ \\
\hline S-ICTP $(\mu \mathrm{g} / \mathrm{l})$ & 14.2 & $7.6-26.3$ & $9.6^{\ddagger}$ & $5.2-16.4$ & $8.2^{\ddagger}$ & $5.5-16.7$ & $18.7^{\dagger} / \mathrm{NS}$ & $10.1-24.9$ & 14.7 & $8.8-28$ \\
\hline S-IGF-I (nmol/I) & $23^{*}$ & $3-37$ & $37^{\ddagger}$ & $21-85$ & $37^{\ddagger}$ & $3-72$ & $27^{\dagger} / \mathrm{NS}$ & $16-50$ & 29 & $13-54$ \\
\hline S-SHBG (nmol/l) & 54 & $22-180$ & $35^{\ddagger}$ & $14-84$ & $28^{\ddagger}$ & $11-108$ & $66 / \mathrm{NS}$ & $22-179$ & 87 & $15-167$ \\
\hline
\end{tabular}

${ }^{\star} P<0.05$ (compared with controls). ${ }^{\dagger} P<0.05$ (compared with pre-treatment levels). ${ }^{\ddagger} P<0.001$ (compared with pre-treatment levels). 
children with active IBD and children with IBD in remission (Table 2). During steroid treatment, SHBG levels declined by $40 \%$ and after therapy returned to pre-treatment levels. In active IBD, SHBG correlated positively with PINP $(r=0.438, n=22, P<0.05)$ and inversely with serum IGF-I $(r=-0.585, n=22$, $P<0.01)$. By contrast to IGF-I levels, there was an inverse correlation between SHBG and body measures: weight $(r=-0.686, n=22, P<0.001)$, height $(r=-0.746, n=22, \quad P<0.001)$ and BMI $(r=-0.424, n=22, P<0.05)$.

\section{The clinical course, glucocorticoid treatment and GBA levels}

Before the onset of the glucocorticoid treatment in this study, $11 / 22$ patients with active IBD had previously received steroid therapy, either with prednisolone or budesonide. The median wash-out period of the previous steroid therapy was 4 (1-25) months. After the start of peroral glucocorticoid, no therapeutic changes were made before the first control visit at 2 weeks of glucocorticoid treatment. At 2 weeks, the median cumulative dose of prednisolone was $14 \mathrm{mg} / \mathrm{kg}$. GBA changed along with the glucocorticoid therapy as previously reported $(19,20)$. The median duration of the steroid therapy was $6(2-29)$ months but the cumulative doses during the total treatment period were not registered. Post-treatment samples were obtained after a median of 1.5 (1-3) months. Of the $21 / 22$ patients who were tapered off steroids, 18 were in clinical remission at this time point and the markers of disease activity had decreased from pre-treatment values, faecal calprotectin from 1610 (23-11 512) to $844(70-2920) \mu \mathrm{g} / \mathrm{g}(n=14, P=\mathrm{NS})$ and ESR from 29 $(6-81)$ to $13(4-50) \mathrm{mm} / \mathrm{h}(n=18, P<0.01)$.

\section{Discussion}

We found that the serum PINP levels reflecting bone formation were significantly lower in patients with active IBD before treatment as compared with IBD controls in clinical remission, a phenomenon potentially attributable to the inhibitory effect of the inflammatory cytokines on bone formation (22). This is supported by the negative correlation between ESR/faecal calprotectin and serum PINP levels in our study and the fact that steroid-naive IBD patients may present with osteoporosis $(23,24)$. Additionally, in active disease other factors such as nutrition or physical inactivity leading to reduced loading of the bones may also contribute to the low PINP levels (25). During the glucocorticoid therapy serum PINP levels decreased further, and after treatment were significantly higher than at baseline. Thus, glucocorticoids appear to affect bone formation in a biphasic fashion with an acute suppressive effect during high circulating GBA levels
(19), followed by an increased rate of bone formation after the cessation of the glucocorticoid therapy.

Pre-treatment serum PINP and SHBG correlated positively, suggesting a possible link between SHBG and osteoblast function in paediatric patients with IBD. A positive correlation between SHBG and the markers of bone metabolism has been reported earlier in young men (17). As SHBG is negatively regulated by the GH/IGF-I system, it is yet to be defined whether the SHBG levels merely mirror their effect on bone metabolism or possibly act directly, for example through the putative SHBG receptor (26).

In children the study protocols have not specifically addressed the change of IGF-I levels during steroid therapy $(25,27)$. As IGF-I levels parallel the markers of bone metabolism in adults, however, we hypothesized that this could be seen also in paediatric IBD patients (17). This was not the case, as serum IGF-I levels did not correlate with the markers of bone formation or resorption either before, during, or after the glucocorticoid treatment. Before therapy the IGF-I levels were low in children with active IBD, consistent with previous studies $(25,27)$. During the glucocorticoid therapy serum IGF-I levels showed an upward trend completely opposite to other markers related to bone turnover and declined only after the cessation of the steroid. These changes could be explained by the anti-inflammatory effect of the steroid or by improved nutritional status (28). However, the exact mechanism of action through which steroids increase the IGF-I levels is still to be defined (29).

Serum ICTP levels reflecting bone resorption displayed a steep decline during the first weeks of steroid therapy, suggesting decreased bone degradation. In literature, the reported effects of glucocorticoids on bone resorption are inconsistent. Some authors have shown glucocorticoid-induced inhibition of bone resorption in vitro and no change or a downward trend in the markers of bone resorption in vivo $(30,31)$. Others have reported prolonged survival of osteoclasts and increased expression of osteoclastogenesis-promoting agents in response to glucocorticoids $(32,33)$. However, prolonged glucocorticoid administration is associated with a risk of osteoporosis (34), a finding reflecting a negative net balance in collagen turnover.

The limitations of this study are the relatively small sample size and somewhat heterogenous study group with regard to aetiology of IBD. Thus, our results might be best applied to paediatric patients with UC. Data on the vitamin-D status and calcium intake of these patients might have been informative with respect to the bone marker levels; unfortunately these data were not available. Our focus was on the acute effects that glucocorticoids have on bone marker levels and therefore the patients were followed-up only for a couple of weeks, a time period too short to detect clinically significant changes in growth velocity. However, the results were compared with pubertal stage matched 
patients with IBD in remission. Despite these limitations, our results show clear impairment of bone turnover during glucocorticoid therapy.

In conclusion, our study shows that bone formation in children with active IBD is compromised but remission is characterized by an increase in bone turnover and metabolism. Administering systemic glucocorticoids to paediatric IBD patients has an acute suppressing effect on bone turnover and metabolism that persists until the withdrawal of the steroid.

\section{Declaration of interest}

The authors declare that there is no conflict of interest that could be perceived as prejudicing the impartiality of the research reported.

\section{Funding}

This work was supported by grants from the Finnish Cultural Foundation, The Emil Aaltonen Foundation, the Helsinki University Central Hospital Grant, the Finnish Paediatric Research Foundation and the Päivikki and Sakari Sohlberg Foundation.

\section{Acknowledgements}

Ms Sari Honkanen, Ms Minna Kuurne-Koivisto, Ms Anne Ramstadt, Ms Katja Kiviniemi and Ms Sari Lindén are thanked for their excellent assistance.

\section{References}

1 Truelove SC \& Witts LJ. Cortisone in ulcerative colitis; final report on a therapeutic trial. BMJ 195529 1041-1048.

2 Diefenbach KA \& Breuer CK. Pediatric inflammatory bowel disease. World Journal of Gastroenterology 200628 3204-3212.

3 Farrell RJ \& Kelleher D. Glucocorticoid resistance in inflammatory bowel disease. Journal of Endocrinology $2003178339-346$.

4 Hyams J, Markowitz J, Lerer T, Griffiths A, Mack D, Bousvaros A, Otley A, Evans J, Pfefferkorn M, Rosh J, Rothbaum R, Kugathasan S, Mezoff A, Wyllie R, Tolia V, delRosario JF, Moyer MS, Oliva-Hemker M, Leleiko N \& Pediatric Inflammatory Bowel Disease Collaborative Research Group. The natural history of corticosteroid therapy for ulcerative colitis in children. Clinical Gastroenterology and Hepatology 20064 1118-1123.

5 Donn R, Berry A, Stevens A, Farrow S, Betts J, Stevens R, Clayton C, Wang J, Warnock L, Worthington J, Scott L, Graham S \& Ray D. Use of gene expression profiling to identify a novel glucocorticoid sensitivity determining gene, BMPRII. FASEB Journal 200721 402-414.

6 Schacke H, Docke WD \& Asadullah K. Mechanisms involved in the side effects of glucocorticoids. Pharmacology and Therapeutics 2002 $9623-43$.

7 Lucarelli S, Borrelli O, Paganelli M, Capocaccia P, Frediani T, Ferri F \& Cucchiara S. Vertebral fractures and increased sensitivity to corticosteroids in a child with ulcerative colitis: successful use of pamidronate. Journal of Pediatric Gastroenterology and Nutrition $200643533-535$.

8 Sorva R, Tähtelä R, Turpeinen M, Juntunen-Backman K, Haahtela T, Risteli L, Risteli J \& Sorva A. Changes in bone markers in children with asthma during inhaled budesonide and nedocromil treatments. Acta Paediatrica 199685 1176-1180.

9 Crofton PM, Ahmed SF, Wade JC, Stephen R, Elmlinger MW, Ranke MB, Kelnar CJ \& Wallace WH. Effects of intensive chemotherapy on bone and collagen turnover and the growth hormone axis in children with acute lymphoblastic leukemia. Journal of Clinical Endocrinology and Metabolism $1998 \mathbf{8 3}$ 3121-3129.

10 Melkko J, Kauppila S, Niemi S, Risteli L, Haukipuro K, Jukkola A \& Risteli J. Immunoassay for intact amino-terminal propeptide of human type I procollagen. Clinical Chemistry $1996 \mathbf{4 2} 947-954$.

11 Melkko J, Niemi S, Risteli L \& Risteli J. Radioimmunoassay of the carboxyterminal propeptide of human type I procollagen. Clinical Chemistry 199036 1328-1332.

12 Risteli J, Elomaa I, Niemi S, Novamo A \& Risteli L. Radioimmunoassay for the pyridinoline cross-linked carboxy-terminal telopeptide of type I collagen: a new serum marker of bone collagen degradation. Clinical Chemistry $199339635-640$.

13 Govoni KE, Baylink DJ \& Mohan S. The multi-functional role of insulin-like growth factor binding proteins in bone. Pediatric Nephrology 200520 261-268.

14 Ueland T. GH/IGF-I and bone resorption in vivo and in vitro. European Journal of Endocrinology 2005152 327-332.

15 Yakar S, Rosen CJ, Beamer WG, Ackert-Bicknell CL, Wu Y, Liu JL, Ooi GT, Setser J, Frystyk J, Boisclair YR \& LeRoith D. Circulating levels of IGF-I directly regulate bone growth and density. Journal of Clinical Investigation $2002 \mathbf{1 1 0} 771-781$.

16 Belgorosky A, Martinez A, Domene H, Heinrich JJ, Bergada C \& Rivarola MA. High serum sex hormone-binding globulin (SHBG) and low serum non-SHBG-bound testosterone in boys with idiopathic hypopituitarism: effect of recombinant human growth hormone treatment. Journal of Clinical Endocrinology and Metabolism 198765 1107-1111.

17 Välimäki VV, Alfthan H, Ivaska KK, Löyttyniemi E, Pettersson K, Stenman UH \& Välimäki MJ. Serum estradiol, testosterone, and sex hormone-binding globulin as regulators of peak bone mass and bone turnover rate in young Finnish men. Journal of Clinical Endocrinology and Metabolism 200489 3785-3789.

18 Lennard-Jones JE. Classification of inflammatory bowel disease. Scandinavian Journal of Gastroenterology 1989170 S2-S6.

19 Vihinen MK, Raivio T, Verkasalo M, Janne OA \& Kolho K-L. Circulating glucocorticoid bioactivity during peroral glucocorticoid treatment in children and adolescents with inflammatory bowel disease. Journal of Clinical Gastroenterology 200842 1017-1024.

20 Vihinen MK, Kolho K-L, Janne OA, Andersson S \& Raivio T. Circulating adiponectin as a marker for glucocorticoid-related side effects in children and adolescents with inflammatory bowel disease. Journal of Pediatric Gastroenterology and Nutrition, (In Press).

21 Kolho KL, Raivio T, Lindahl H \& Savilahti E. Fecal calprotectin remains high during glucocorticoid therapy in children with inflammatory bowel disease. Scandinavian Journal of Gastroenterology $200641720-725$.

22 Clowes JA, Riggs BL \& Khosla S. The role of the immune system in the pathophysiology of osteoporosis. Immunological Reviews 2005 208 207-227.

23 Paganelli M, Albanese C, Borrelli O, Civitelli F, Canitano N, Viola F, Passariello $\mathrm{R} \&$ Cucchiara $\mathrm{S}$. Inflammation is the main determinant of low bone mineral density in pediatric inflammatory bowel disease. Inflammatory Bowel Diseases $2007 \mathbf{1 3}$ 416-423.

24 Walther F, Fusch C, Radke M, Beckert S \& Findeisen A. Osteoporosis in pediatric patients suffering from chronic inflammatory bowel disease with and without steroid treatment. Journal of Pediatric Gastroenterology and Nutrition $2006 \mathbf{4 3} 42-51$.

25 Sylvester FA, Wyzga N, Hyams JS, Davis PM, Lerer T, Vance K, Hawker G \& Griffiths AM. Natural history of bone metabolism and bone mineral density in children with inflammatory bowel disease. Inflammatory Bowel Diseases 200713 42-50.

26 Kahn SM, Hryb DJ, Nakhla AM, Romas NA \& Rosner W. Sex hormone-binding globulin is synthesized in target cells. Journal of Endocrinology $2002175113-120$

27 Corkins MR, Gohil AD \& Fitzgerald JF. The insulin-like growth factor axis in children with inflammatory bowel disease. Journal of Pediatric Gastroenterology and Nutrition $200336228-234$.

28 Eivindson M, Grønbaek H, Flyvbjerg A, Frystyk J, ZimmermannNielsen E \& Dahlerup JF. The insulin-like growth factor (IGF) 
system in active ulcerative colitis and Crohn's disease: relations to disease activity and corticosteroid treatment. Growth Hormone and IGF Research 200717 33-40.

29 Clemmons DR. Clinical utility of measurements of insulin-like growth factor 1. Nature Clinical Practice. Endocrinology and Metabolism 20062 436-446.

30 Dempster DW, Moonga BS, Stein LS, Horbert WR \& Antakly T. Glucocorticoids inhibit bone resorption by isolated rat osteoclasts by enhancing apoptosis. Journal of Endocrinology $1997 \mathbf{1 5 4}$ 397-406.

31 Ton FN, Gunawardene SC, Lee H \& Neer RM. Effects of low-dose prednisone on bone metabolism. Journal of Bone and Mineral Research 200520 464-470.

32 Weinstein RS, Chen JR, Powers CC, Stewart SA, Landes RD, Bellido T, Jilka RL, Parfitt AM \& Manolagas SC. Promotion of osteoclast survival and antagonism of bisphosphonate-induced osteoclast apoptosis by glucocorticoids. Journal of Clinical Investigation 2002109 1041-1048.

33 Kwan Tat S, Padrines M, Théoleyre S, Heymann D \& Fortun Y. IL-6, RANKL, TNF- $\alpha /$ IL-1: interrelations in bone resorption pathophysiology. Cytokine and Growth Factor Reviews 200415 49-60.

34 Semeao EJ, Jawad AF, Stouffer NO, Zemel BS, Piccoli DA \& Stallings VA. Risk factors for low bone mineral density in children and young adults with Crohn's disease. Journal of Pediatrics 1999 135 593-600.

Received 13 August 2008

Accepted 23 August 2008 